\title{
Endovascular brachytherapy for extensive right-heart and pulmonary artery sarcoma - a case report
}

\author{
Gal Aviel, MD*, Amichay Meirovitz, MD²*, David Planer, MD³, Dotan Cohen, MD4, Dan Gilon, MD³, Jon Feldman, PhD², \\ Oz M. Shapira, MDl \\ 'Department of Cardiothoracic Surgery, Hadassah Hebrew University Medical Center, Jerusalem, Israel, ${ }^{2}$ Department of Oncology, Hadassah \\ Hebrew University Medical Center, Jerusalem, Israel, ${ }^{3}$ Department of Cardiology, Hadassah Hebrew University Medical Center, Jerusalem, \\ Israel, ${ }^{4}$ Department of Radiology, Hadassah Hebrew University Medical Center, Jerusalem, Israel \\ ${ }^{*}$ Gal Aviel and Amichay Meirovitz contributed equally to this work.
}

\begin{abstract}
Purpose: Primary sarcoma of the heart is a rare but devastating tumor. Median survival with conventional treatment is 8-12 months. When resection is not feasible, patients often succumb to heart failure secondary to obstruction of blood flow, valve dysfunction, chamber compression or conduction abnormalities. Palliative treatment options include systemic chemotherapy and external beam irradiation. We herein describe a novel technique using endovascular brachytherapy, aiming at reducing tumor mass, alleviating right ventricular pressure overload and at the same time keeping the option of $\mathrm{R} 0$ resection viable.

Material and methods: A 35-year-old man was diagnosed with a non-resectable high-grade intimal sarcoma of the right ventricle (RV), main pulmonary artery (PA) and right PA. After three cycles of doxorubicin and ifosfamide, the patient's symptoms of right heart failure worsened. Imaging documented tumor progression and supra-systemic pulmonary artery pressure. Through a trans-femoral venous access, a brachytherapy sleeve was placed in the RV and main and right PA. A dose of $20 \mathrm{~Gy}$ was delivered over a period of ten minutes.

Results: The patient had an uneventful course and was discharged home 24 hours after the procedure. Ten months after brachytherapy, repeat imaging demonstrated a significant reduction in tumor volume and an increase in pulmonary artery cross-sectional area with a marked reduction of pulmonary artery pressure, leading to a complete resolution of heart failure symptoms.

Conclusions: Endovascular brachytherapy is a novel, safe and effective therapeutic modality for non-resectable primary cardiac sarcomas either for palliation of obstruction, or tumor mass reduction to allow complete resection.

Key words: brachytherapy, right heart sarcoma, pulmonary artery sarcoma.

\section{Purpose}

Primary sarcomas of the heart are rare tumors with a median survival of 8-12 months [1]. Multimodality treatment that includes complete resection effectively prolongs survival [1,2]. Patients with unresectable tumors often succumb to heart failure due to obstructive lesions of the heart. Pulmonary hypertension is an indicator of poor prognosis [3]. We describe a patient with unresectable right heart sarcoma successfully treated with endovascular brachytherapy for a right-heart and pulmonary artery (PA) sarcoma.

\section{Case report}

A 35-year-old man presented with a three-month history of progressive dyspnea and palpitations. Work- up revealed a mass in the right ventricle (RV), involving the tricuspid valve, partially obstructing the outflow tract, extending to the main and right pulmonary arteries, causing significant luminal stenosis (Figure 1). Right ventricle tumor volume by computed tomography (CT, Philips iCT256 [Philips, Surrey, UK], gaited, $0.6 \mathrm{~mm}$ slices, $120 \mathrm{kV}, 600 \mathrm{mAs}$ ) was $16.6 \mathrm{ml}$. Main pulmonary artery (MPA) cross-sectional area was $3.15 \mathrm{~cm}^{2}$, and maximal wall thickness was $8.3 \mathrm{~mm}$. Right PA cross-sectional area was $1.77 \mathrm{~cm}^{2}$ and maximal wall thickness was $8.0 \mathrm{~mm}$ (Figure 2). Pulmonary artery pressure assessed by 2D echocardiography (Philips EPIQ7, Philips, Surrey, UK) was supra-systemic. Endomyocardial biopsy confirmed the diagnosis of a high-grade intimal sarcoma.

After three cycles of doxorubicin and ifosfamide, the patient's symptoms of right heart failure worsened. Im- 

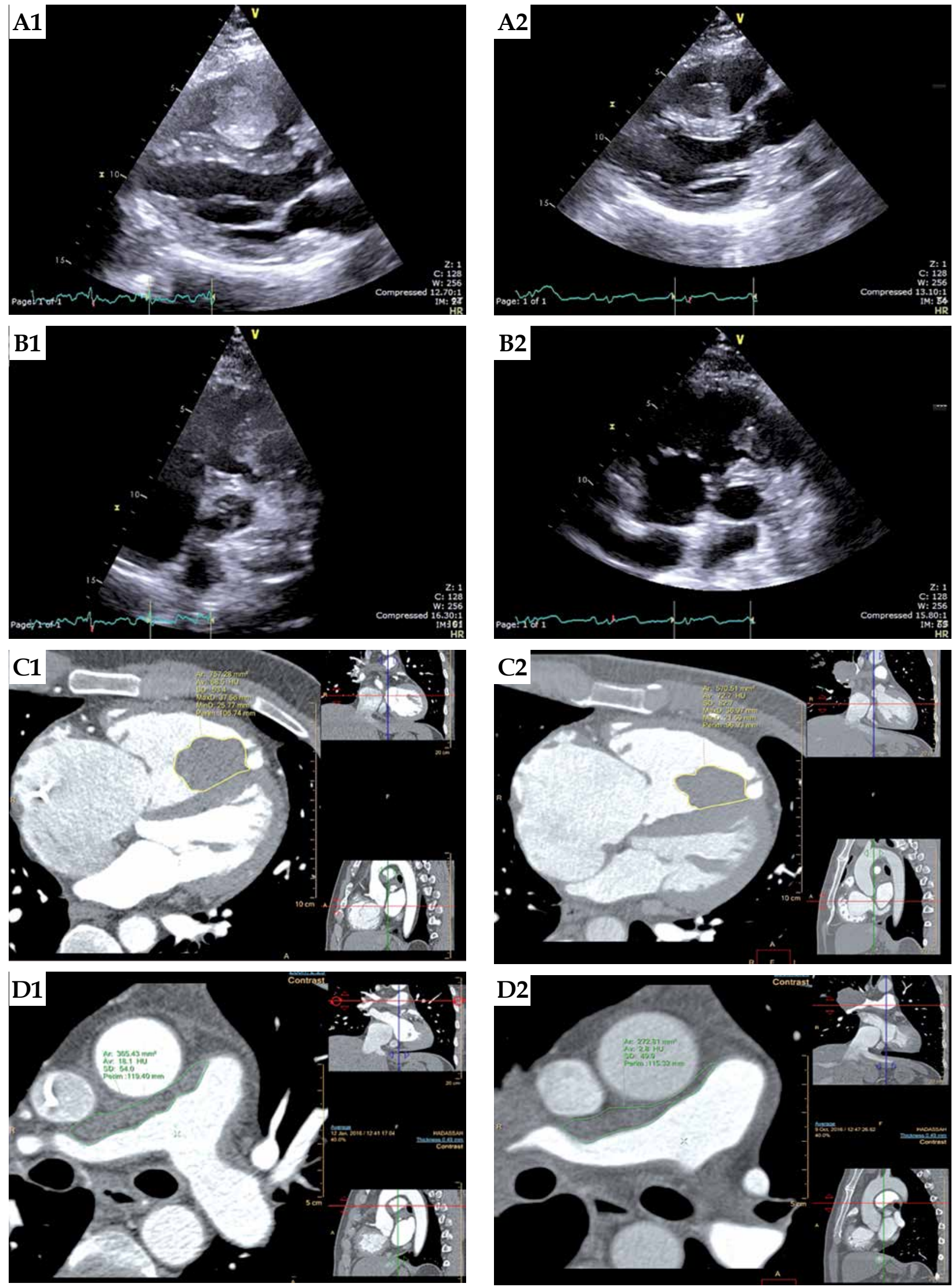

Fig. 1. A, C) The right ventricular mass on transthoracic echocardiography and computed tomography before and after therapy (A1 and A2, C1 and C2, respectively). B, D) The mass in the right ventricular outflow tract, extending to the right pulmonary artery on TTE and CT, before and after therapy (B1 and B2, D1 and D2, respectively). Following endovascular brachytherapy, a $26 \%$ decrease in volume is apparent in the pulmonary artery mass and a $40 \%$ decrease in RV mass volume 

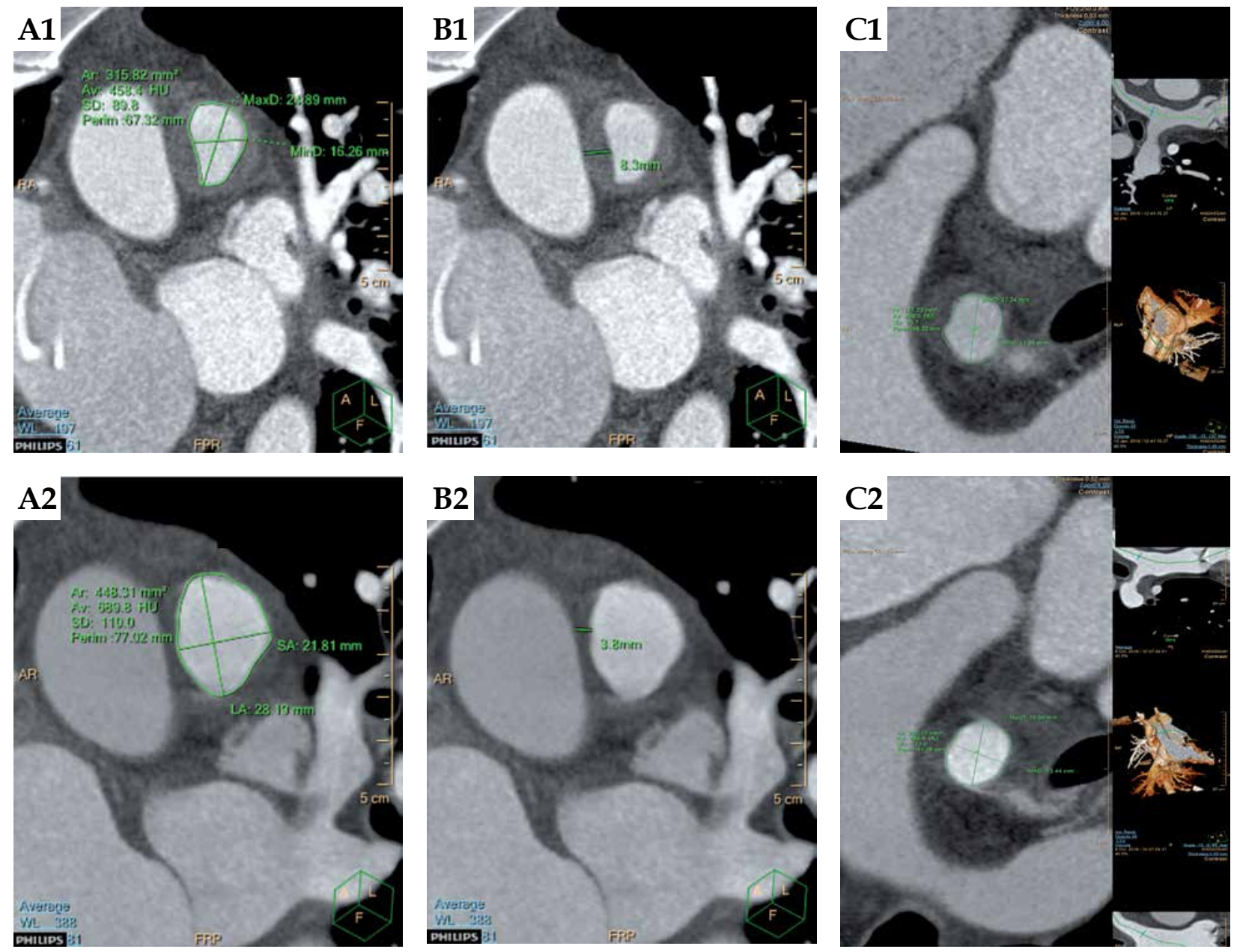

Fig. 2. A) CT scan of the cross-sectional area of the pulmonary trunk before and after treatment (A1 and $\mathbf{A 2}$, respectively).

B) The MPA maximal wall thickness before and after treatment (B1 and B2, respectively). A 42\% increase in cross-sectional area and a 55\% decrease in wall thickness are demonstrated following brachytherapy. C) RPA cross sectional area and maximal wall thickness before treatment $\left(1.77 \mathrm{~cm}^{2}\right.$ and $8.0 \mathrm{~mm}$ respectively, $\left.\mathbf{C} 1\right)$ and afterwards $\left(2.0 \mathrm{~cm}^{2}\right.$ and $\left.6.7 \mathrm{~mm}, \mathbf{C} 2\right)$

aging documented tumor progression. To minimize the effects of external beam irradiation on the heart and keep the option of a radical R0 resection viable, we elected to utilize endovascular brachytherapy.

\section{Material and methods}

This was a one-time treatment of high-dose-rate (HDR) brachytherapy. No endovascular devices, such as stents or seeds, were implanted. Given the novelty of the procedure, detailed informed consent was obtained. The initial procedure was performed in the cardiac catheterization laboratory. Through a transfemoral venous access, a soft guidewire was placed in the RV and the right PA. The patient was then transferred to the radiotherapy suite. A brachytherapy sleeve was inserted over the wire and anchored to a distal PA branch to prevent migration. A $14 \mathrm{~cm}, 6 \mathrm{~F}$ demonstration catheter was inserted through the sleeve for dosage calculation and a CT simulation was performed (Figure 3). The CT plan was transferred to the PLATO planning system (Elekta AB, Stockholm, Sweden) and a dose of $20 \mathrm{~Gy}$ was planned to the $0.7 \mathrm{~cm}$ isodose line. We chose the radial dimensions based on the CT simulation, the tumor thickness within the RV and PA, and the position of the catheter. The $\mathrm{V}_{100}, \mathrm{~V}_{150}$ and $\mathrm{V}_{200}$ for the PA and RV were $51 \%, 34 \%, 23 \%$ and $28 \%, 16 \%$, $10 \%$, respectively (Figure 3 ). Since our primary goal was to maintain vessel patency, tumor volume conformality was suboptimal. The irradiation was delivered over a period of ten minutes using a ${ }^{192}$ Ir source (microSelectron, Elekta AB, Stockholm, Sweden). The delivery system was removed at the end of the procedure. We aimed to reduce the irradiation to the right heart itself, the tricuspid valve and the esophagus to a minimum (Figure 4).

\section{Results}

The patient had an uneventful course and was discharged home 24 hours after the procedure. Ten months after brachytherapy, repeat CT angiography demonstrated a $40 \%$ reduction in the RV tumor volume (Figure $1 \mathrm{~A}, \mathrm{C})$, a $42 \%$ increase in MPA cross-sectional area, and a $55 \%$ reduction in its wall thickness (Figure 2A,B). Symptoms of heart failure completely resolved, parallel 

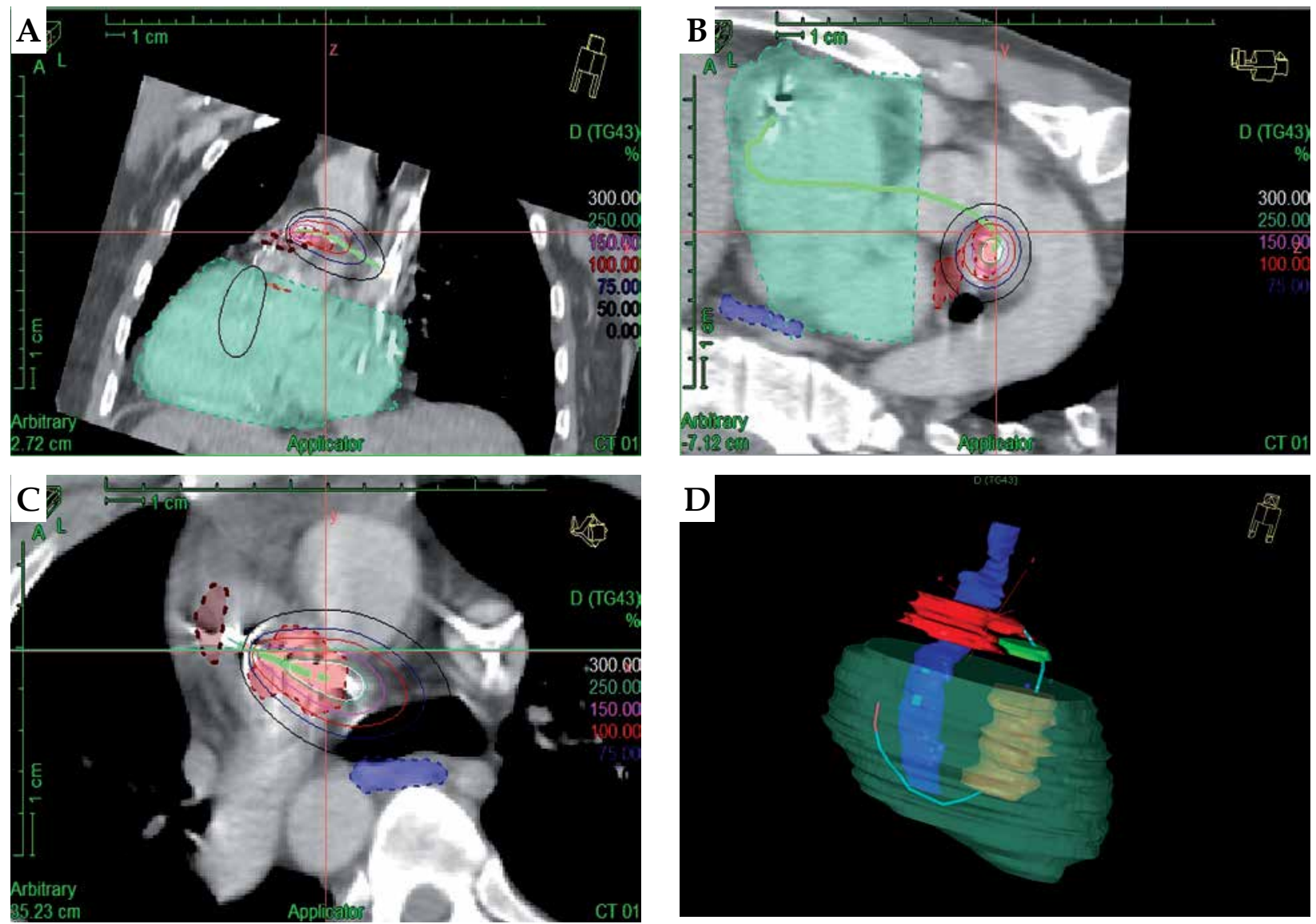

Fig. 3. CT scan-based brachytherapy plan. A-C) The isodose levels around the delivery catheter at different projections. D) The 3D imaging of the delivery catheter and the relevant structures. Pulmonary artery tumor mass - red; right ventricle free wall green; right ventricular tumor mass - yellow; esophagus - dark blue

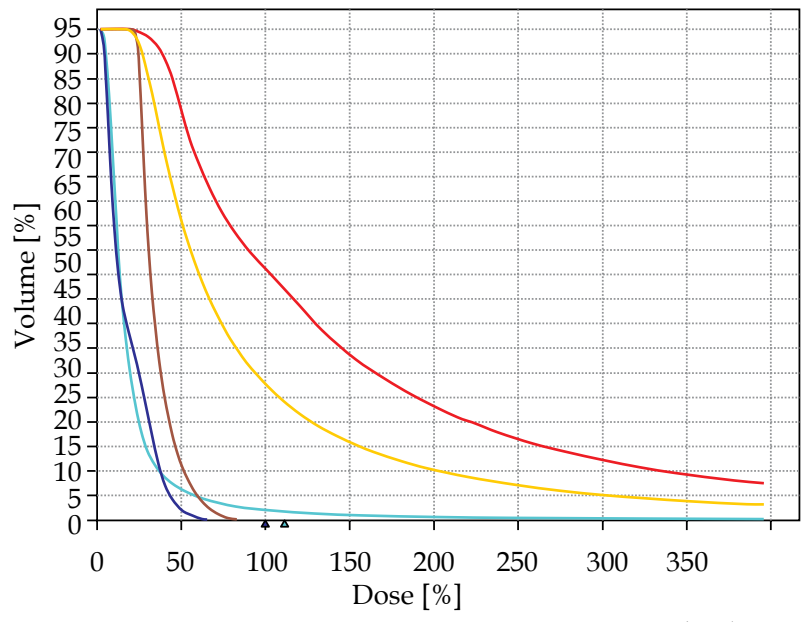

- PA tumor - RV tumor - Tricuspid valve — RV free wall — Esophagus

Fig. 4. Dose-volume histograms. The irradiation doses delivered to the right ventricle (RV) free wall, tricuspid valve and the esophagus were substantially lower than those delivered to the primary targets - the RV and pulmonary artery (PA) tumor masses. PA tumor mass - red; right ventricle tumor mass - yellow; tricuspid valve - brown; right ventricular free wall - light blue; esophagus - dark blue to the radiological response. The patient was reluctant to receive additional chemotherapy of any kind. The patient succumbed to a widespread metastatic spread 38 months after the initial diagnosis with slightly elevated pulmonary artery pressures and no side-effects related to the brachytherapy.

\section{Discussion}

We describe a case of a young patient presenting with obstructive right-heart sarcoma that did not respond to chemotherapy, and was deemed inoperable due to extensive involvement of the right ventricle and pulmonary arteries. The prognosis in this setting is exceedingly poor $[1,4,5]$. The remaining therapeutic options are limited, and include second- and third-line chemotherapy regimens and external beam irradiation. The patient declined chemotherapy due to the intense side-effects he had suffered.

In patients with primary sarcoma, the heart is usually more sensitive to irradiation than the tumor, therefore precluding normal-dose radiotherapy [5]. Advanced imaging including positron emission tomography, CT and magnetic resonance imaging that allow precise treatment planning make external beam irradiation more feasible and tolerable [6]. However, it is still reserved for patients who are not candidates for radical curative surgery. 
We elected to utilize endovascular brachytherapy as a novel therapeutic approach, aiming to reduce tumor load, restore flow in the PA, alleviate symptoms of heart failure secondary to right ventricle pressure overload, and at the same time keep the option of radical surgery by avoiding high-dose external beam irradiation to the heart. We achieved a $40 \%$ reduction in RV tumor volume, a $42 \%$ increase in MPA cross-sectional area and complete resolution of heart failure symptoms. We achieved the therapeutic goal with essentially no side-effects since adjacent structures such as the right ventricular free wall, tricuspid valve and the esophagus received a substantially lower dose of irradiation than the primary tumor. The patient was reluctant to receive additional chemotherapy. Thus, despite the favorable response to brachytherapy, the extent of the tumor was such that a complete (R0) resection was not feasible.

Endoluminal brachytherapy is infrequently used for cancer treatment. Only a handful of reports describe the utility of intra-venous stent and ${ }^{125}$ I seed implantation in patients with cancer-associated portal vein or peripheral vein tumor occlusion $[7,8]$.

To the best of our knowledge this is the first report of using endovascular brachytherapy for primary rightheart and PA sarcoma. The advantages of this novel therapeutic approach include a short, one-time treatment delivery in contrast to multiple treatment sessions, rapid tumor regression alleviating hemodynamically significant obstruction and avoiding implantation of a thrombogenic foreign body. Precise, localized targeting allows delivery of high-dose irradiation dose to the tumor, avoiding side-effects on adjacent heart structures, frequently observed after external beam irradiation to the constantly moving heart, and often precluding subsequent definitive surgery $[5,6]$. The main potential hazard of this approach is vascular perforation during the initial procedure or at the time of maximal treatment effect.

In conclusion, endovascular brachytherapy is a novel, safe and effective therapeutic modality for unresectable primary cardiac sarcomas, either for palliation of obstruction, or tumor mass reduction to allow complete resection.

\section{Disclosure}

The authors report no conflict of interest.

\section{References}

1. Yanagawa B, Mazine A, Barker CM et al. Surgery for tumors of the heart. Semin Thorac Surg 2018; 30: 385-397.

2. Saleh WKA, Ramlawi B, Shapira OM et al. Improved outcomes with the evolution of a neoadjuvant chemotherapy approach to right heart sarcoma. Ann Thorac Surg 2017; 104: 90-96.

3. Lee SD, Li CM, Kim WS et al. Clinical characteristics and treatment outcomes of primary pulmonary artery Sarcoma in Korea. J Korean Med Sci 2016; 31: 1755-1760.

4. Morreau SP, Haydock DA. Prolonged survival of pulmonary artery sarcoma after aggressive surgical resection. Ann Thorac Surg 2017; 103: e21-23.

5. Reardon MJ, Walkes JC, Benjamin R et al. Therapy insight: malignant primary cardiac tumors. Nat Clin Pract Cardiovasc Med 2006; 3: 548-553.
6. Elsayad K, Scobioala S, Kriz J et al. Advances in image-guided radiation therapy for primary cardiac angiosarcoma: The role of PET-CT and MRI. Oncol Res Treat 2016; 39: 290-294.

7. Zhang ZH, Liu QX, Zhang $W$ et al. Combined endovascular brachytherapy, sorafenib, and transarterial chemoembolization therapy for hepatocellular carcinoma patients with portal vein tumor thrombus. World J Gastroenterol 2017; 23: 7735-7745.

8. Gong $\mathrm{M}$, Song J, He X et al. Intraluminal brachytherapy using self-expandable stent loaded with linear-Idonie- 125 seeds in patient with reiterative recurrence of cancer-associated vein obstrauction: A case report with 3 months of follow-up. Vasc Endovasc Surg 2018; 52: 463-468. 